\title{
Estimating number of cases and spread of coronavirus disease (COVID-19) using critical care admissions, United Kingdom, February to March 2020
}

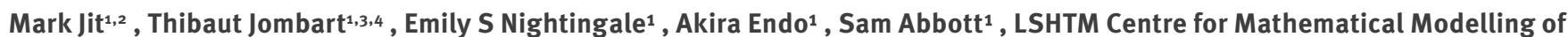

Infectious Diseases COVID-19 Working Group ${ }^{5}$, W John Edmunds ${ }^{1}$

1. Centre for Mathematical Modelling of Infectious Diseases, Department of Infectious Disease Epidemiology, London School of Hygiene and Tropical Medicine, London, United Kingdom

2. School of Public Health, Li Ka Shing Faculty of Medicine, University of Hong Kong, Hong Kong SAR, China

3. United Kingdom Public Health Rapid Support Team, London, United Kingdom

4. MRC Centre for Global Infectious Disease Analysis, Department of Infectious Disease Epidemiology, School of Public Health, Imperial College London, United Kingdom

5. The members of the LSHTM Centre for Mathematical Modelling of Infectious Diseases COVID-19 Working Group are listed at the end of this article

Correspondence: Mark Jit (mark.jit@lshtm.ac.uk)

Citation style for this article:

Jit Mark, Jombart Thibaut, Nightingale Emily S, Endo Akira , Abbott Sam , LSHTM Centre for Mathematical Modelling of Infectious Diseases COVID-19 Working Group, Edmunds W John. Estimating number of cases and spread of coronavirus disease (COVID-19) using critical care admissions, United Kingdom, February to March 2020. Euro Surveill. 2020;25(18):pii=2000632. https://doi.org/10.2807/1560-7917.ES.2020.25.18.2000632

An exponential growth model was fitted to critical care admissions from two surveillance databases to determine likely coronavirus disease (COVID-19) case numbers, critical care admissions and epidemic growth in the United Kingdom before the national lockdown. We estimate, on 23 March, a median of 114,000 (95\% credible interval ( $\mathrm{Crl})$ : 78,000-173,000) new cases and 258 (95\% Crl: 220-319) new critical care reports, with 527,000 (95\% Crl: 362,000-797,000) cumulative cases since 16 February.

The first reported cases of coronavirus disease (COVID19) in the United Kingdom (UK) were identified in late January 2020 [1]. By 23 March, when a national lockdown prohibiting non-essential movement was announced, reported cases had increased to over 6,000. However, reported cases are probably a small fraction of total infections because most COVID-19 cases are mild or even asymptomatic [2]. Also, the proportion of infections that are reported changes by setting and over time, depending on the intensity of population-based surveillance, testing and contact tracing. However, COVID-19 patients admitted to critical care (highdependency or intensive care units, hereafter (C) offer a more stable indicator, since CC patients suspected of having COVID-19 have always been tested in the UK. We used the two COVID-19 CC surveillance databases available at the start of the epidemic, combined with data on reporting delays and disease severity to determine the likely number of and increase in infected people during the first phase of the COVID-19 epidemic in the UK. This work informed estimates produced by the Scientific Pandemic Influenza Group on Modelling
(SPI-M) to advise the UK's Department for Health and Social Care on its COVID-19 response.

\section{Data sources \\ Two data sources were used for CC case numbers:}

(i) Public Health England (PHE) compiled the First Few Hundred (FF100) database containing virologically confirmed COVID-19 cases in the UK during the first phase of the epidemic [3]. We extracted 'sporadic' cases which were those identified through CC surveillance rather than contact tracing or targeted testing of travellers from high-risk countries, so that our analyses focussed on epidemic spread within the UK rather than including case importation. A few sporadic cases were identified through sentinel primary care testing rather than CC but could not be identified as such; however, there were only three such cases so the numbers were too small to affect our results. Sporadic cases were extracted from 16 February (the earliest date of onset for a sporadic case) to 6 March; cases peaked at six per day on 6 March and then declined, indicating that the FF100 was increasingly incomplete.

(ii) PHE and National Health Service (NHS) England compiled CC cases in the COVID-19 Hospitalisations in England Surveillance System (CHESS) database which were collected on 15-20 March (the seven cases reported on 13 and 14 March were ignored as the system was not fully operational then) [4].

We estimated the age-dependent risk of an infected case being admitted to CC by fitting our models to 


\section{FIGURE}

Model estimates of daily new COVID-19 infections and critical care reports in the initial phase of the epidemic compared with source data, United Kingdom, February-March 2020

\section{A. $\mathrm{FF} 100$}

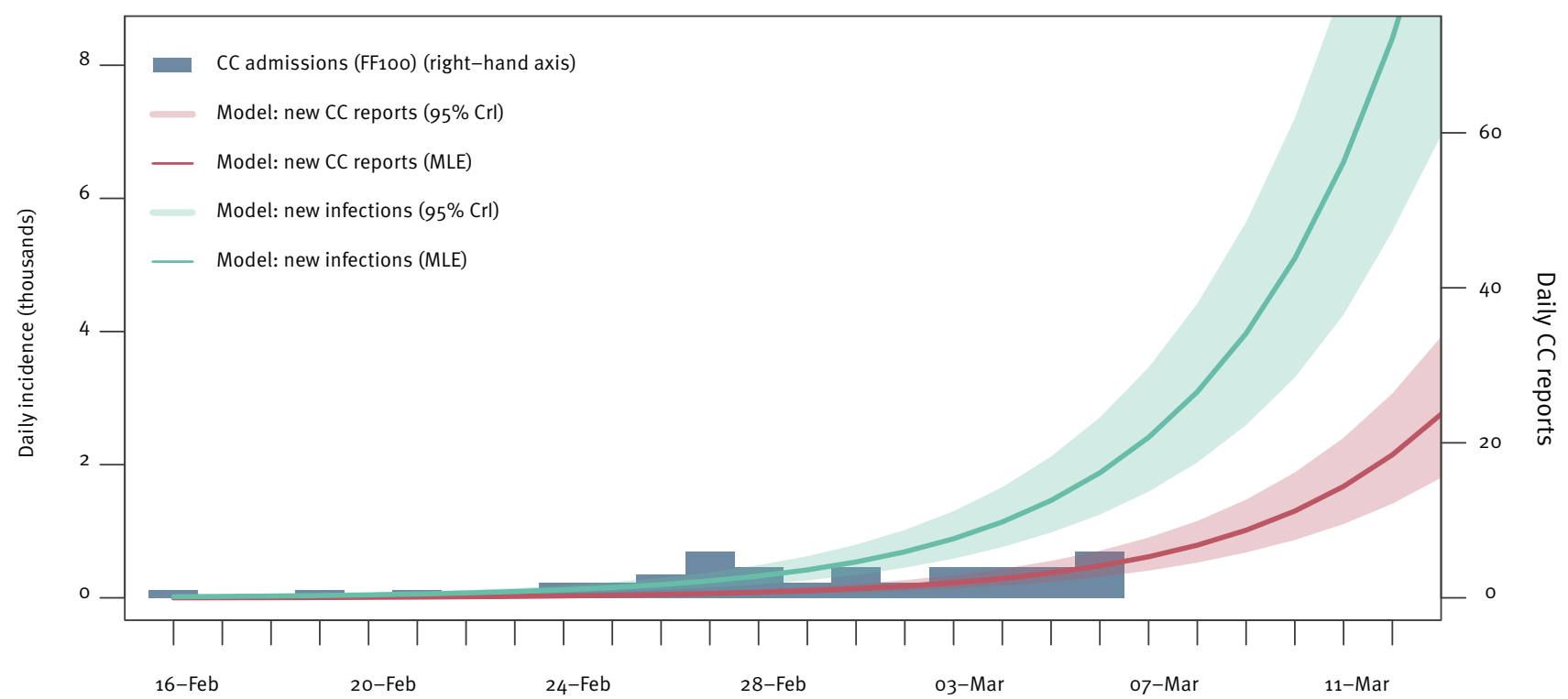

Date

\section{B. CHESS (over longer time scale)}

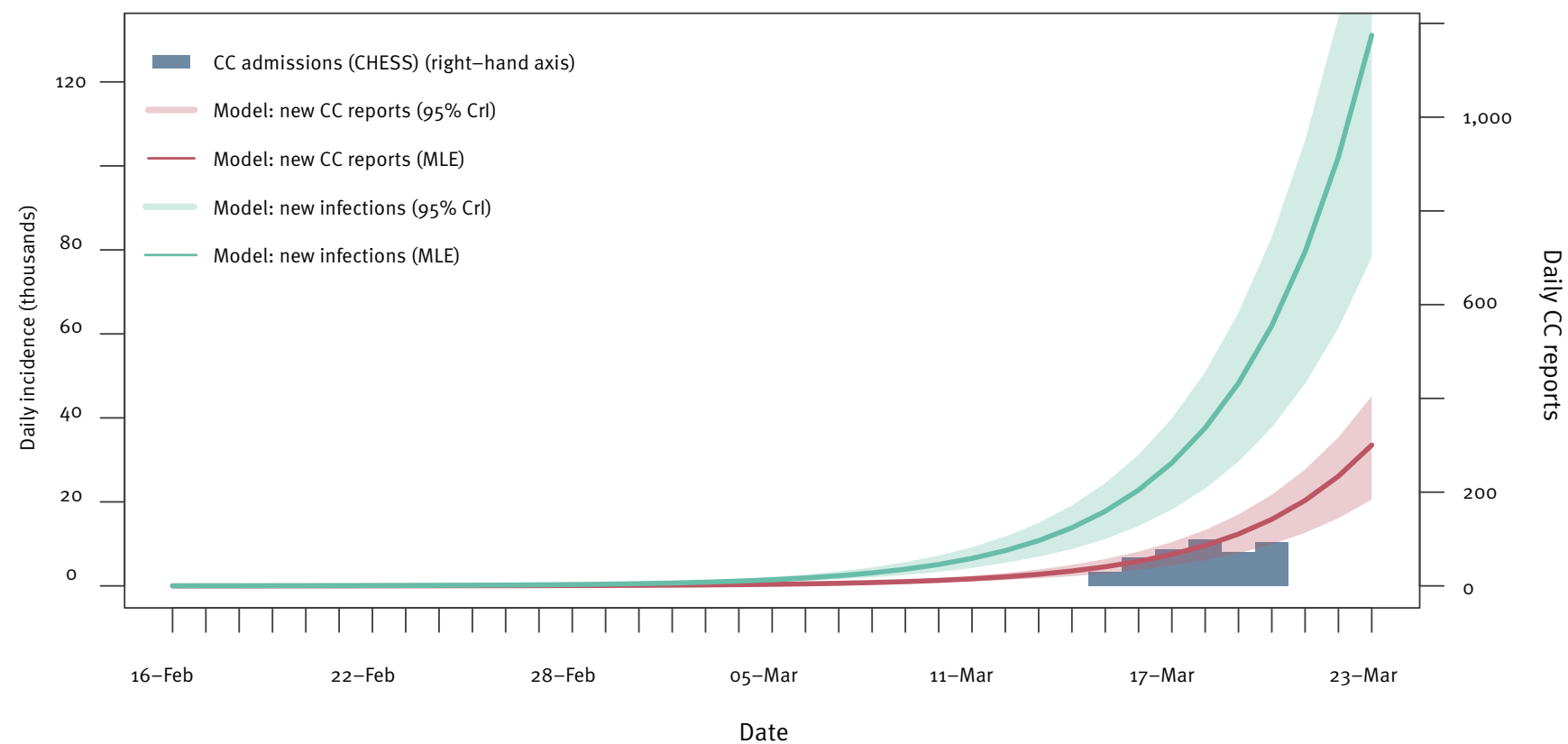

CC: critical care; CHESS: COVID-19 hospitalisations in England surveillance system; FF10o: first few hundred; MLE: maximum likelihood estimate; $95 \% \mathrm{Crl}$ : $95 \%$ credible interval.

The curves corresponding to the MLE are in darker colours. 
analyses of case data from China [5] and the United States (US) [6], with recently released Italian data [7] used in a sensitivity analysis (details in Supplement Chapter 1).

\section{Modelling}

The same model was fitted independently to the incidence of patients admitted to CC based on (i) their date of symptom onset (for FF10o patients) or (ii) their date of admission (for CHESS patients, as date of symptom onset was not reported), using a Poisson likelihood with rate $A e^{B t}$, where $A$ is the initial number of cases on 16 February, $B$ is the growth rate of the epidemic, $t$ is time in days after 16 February and $e$ is the exponential constant (see Supplement Chapter 4). Delays between symptom onset and reporting were accounted for by fitting a gamma distribution to time between symptom onset and CC admission for $\mathrm{FF}_{100}$ patients (see Supplement Chapter 2). When fitting modelled CC admissions to $\mathrm{FF} 100$, incidence was inflated by $18.6 \%$ compared with CC admissions fitted to CHESS, to adjust for the ratio of the population of the UK to that of England (the ratio of reported COVID-19 cases in the UK to that reported in England on 15-23 March would give a similar inflation factor of $19.8 \%$ ).

$A$ and $B$ were sampled from their posterior distributions by using importance sampling; 10,000 parameter sets for both were drawn from uniform distributions between 0 and 1 , and then resampled with replacement at a probability for each sample weighted by the likelihood of that parameter set. Projected CC cases were then divided by age-dependent risks of $\mathrm{CC}$ admission to estimate actual infections in the population (Supplement Chapter 1).

\section{Results}

We estimated that each COVID-19 case admitted to CC reported in FF100 and CHESS corresponded to a median of 124 (95\% credible interval (Crl): 81-11,500) and 120 (95\% Crl: 76-46,600) infected individuals in the population, respectively, based on Chinese and US severity data $[5,6]$.

The Figure shows the number of incident cases estimated on each day between 16 February and 23 March. On 23 March, 114,000 (95\% Crl: 78,000173,000) new cases and 258 (95\% Crl: 220-319) CC reports are estimated to have occurred, with 527,000 (95\% Crl: 362,000-797,000) cumulative cases since 16 February. The best fitting exponential growth rates were consistent with an epidemic doubling time of 2.8 days (95\% Crl: 2.6-3.0). Assuming an exponentially distributed serial interval of 4 days [8] gave an (approximate) reproduction number of 2.0 (95\% Crl: 1.9-2.1). If we assume a longer serial interval of 6 days that may be expected at the start of an epidemic, the reproduction number could be 2.5 ( $95 \% \mathrm{Crl}: 2.4-2.6$ ).

We performed sensitivity analyses on the datasets used for fitting, sensitivity of case detection and reporting, period of validity of the FF10o data and severity of COVID-19. Across the scenarios, the number of new infections on 23 March ranged from 95,000 (95\% Crl: 62,000-182,000) to 143,000 (95\% Crl: 95,000-228,000) (Supplement Chapter 3).

For validation, we compared the median total number of CC admissions from 16 to 23 March (with that period of time chosen to correspond to an average duration of CC stay of 8 days in Wuhan data [9]) with the prevalence of COVID-19 patients in CC on 23 March (i.e. number of patients reported to be in (C) according to NHS England's situation report. Our model projected that 1,200 (95\% Crl: $1,060-1,380$ ) patients were in CC on 23 March, ca $68 \%$ more than the figure of 714 from the situation report when inflated to account for the larger UK population.

\section{Discussion}

Our results suggest that hundreds of thousands of COVID-19 infections had occurred in the UK by the time the national lockdown of 23 March was implemented, with incidence doubling every 2.8 (95\% Crl: 2.6-3.0) days. This suggests that only around $1 \%$ of infections were being detected and reported, since the official case count on 23 March was 6,650 [1]. This provides evidence that strict physical distancing was necessary to prevent health services from being overwhelmed.

However, these figures are still orders of magnitude below the scenarios from Lourenço et al. [10] that suggest that the majority of the UK population (66 million) could have already been infected by that date. The Lourenço scenarios producing these conclusions rely on severity pyramids that are much milder than those observed from Chinese [5], US [6] and Italian [7] data. Our own analyses indicate that severity is indeed a key driver of uncertainty in case estimates but across all scenarios, the majority of the UK population remained uninfected, and hence timely interventions to reduce physical contact could have a large impact. Importantly, our analyses relied on data from these countries only to establish the proportion of infected people who required critical care (which we expect to vary less between countries with reasonable healthcare access), and not for parameters likely to differ widely between countries such as the proportion of cases which are reported.

Our investigations revealed weaknesses in two early sources of CC surveillance (FF100 and CHESS). In particular, the number of cases reported in the two databases differ markedly, with the FF10o reporting far fewer CC cases (e.g. six cases on 6 March from FF10o compared with 100 on 18 March from CHESS). Our model projections suggest that the FF10o numbers are lower even after accounting for different periods of coverage and delays to reporting. Further analysis also suggested delays between the date of being reported as a case and the date on which a case is actually recorded in the FF100 database which made correcting 
for right-truncation of cases difficult (see Supplement Chapter 2). Also, the proportion of cases estimated to require CC treatment was substantially lower than observed in other countries (and hence the multiplication factors to convert CC cases to infections are higher) because of the young age profile of $\mathrm{CC}$ cases in both FF100 and CHESS. For example, 34\% of CC patients were younger than 45 years in CHESS compared with only $12 \%$ in published US data up to 16 March [6]. This may reflect biases in CHESS reporting, differential propensity to test by age or a higher triage threshold for CC admission of older patients than in the US.

The overall conclusion that COVID-19 daily incidence in the UK on 23 March was in the hundreds of thousands of infections and cumulative incidence around half a million appears to be robust even when challenged by a range of sensitivity analyses to account for different assumptions. Our model was fitted to data from early on in the epidemic (16 February) to just before the national lockdown (20 March). Furthermore, the order of magnitude is the same as data from situation reports that have less granularity but possibly better coverage. Lower numbers in the situation reports may in part reflect decreasing propensity to admit patients as CC beds become filled, in line with triage guidelines issued during the pandemic [9]. In addition, our model may have slightly overestimated cases if their rate of increase had slowed down even before the lockdown started. This highlights the need to triangulate case estimates from multiple surveillance streams whenever possible. Our investigations highlight the usefulness of CC surveillance in understanding epidemic dynamics and informing response measures, and hence the need for timely and complete reporting over the course of the epidemic.

LSHTM Centre for Mathematical Modelling of Infectious Disease COVID-19 working group

Arminder K Deol, Hamish Gibbs, Kathleen O’Reilly, Graham Medley, Joel Hellewell, Alicia Rosello, Billy J Quilty, Charlie Diamond, Petra Klepac, Amy Gimma, Rosalind M Eggo, Megan Auzenbergs, Samuel Clifford, Gwen Knight, Sebastian Funk, Anna Foss, Fiona Sun, Jon C Emery, Kiesha Prem, Yang Liu, Kevin van Zandvoort, Christopher I Jarvis, James D Munday, Adam J Kucharski, Carl A B Pearson, Timothy W Russell, Nikos I Bosse, Stefan Flasche, Rein M G J Houben, Simon R Procter, Nicholas G. Davies.

\section{Acknowledgements}

We would like to acknowledge the other members of the London School of Hygiene and Tropical Medicine COVID-19 modelling group, who contributed to this work. Their funding sources are as follows: Hamish Gibbs (NIHR ITCRZ 03010), Kathleen O'Reilly (BMGF OPP1191821), Joel Hellewell (Wellcome Trust 210758/Z/18/Z), Alicia Rosello (NIHR PROD-1017-20002), Billy Quilty (NIHR 16/137/109), Charlie Diamond (NIHR 16/137/109), Petra Klepac (BMGF INV-003174, EU 101003688), Amy Gimma (RCUK/ESRC ES/Po10873/1), Rosalind M Eggo (HDR UK MR/So03975/1), Megan Auzenbergs (BMGF OPP1191821), Samuel Clifford (Wellcome Trust 208812/Z/17/Z), Gwen Knight (MRC MR/P014658/1),
Sebastian Funk (Wellcome Trust 210758/Z/18/Z), Fiona Sun (NIHR 16/137/109), Jon C Emery (ERC \#757699), Kiesha Prem (BMGF INV-003174, EU 101003688), Yang Liu (BMGF INV-003174, NIHR 16/137/109, EU 101003688), Kevin van Zandvoort (R2HC), Christopher I Jarvis (GCRF ES/P010873/1), James D Munday (Wellcome Trust 210758/Z/18/Z), Adam J Kucharski (Wellcome Trust 206250/Z/17/Z), Carl A B Pearson (BMGF OPP1184344), Timothy W Russell (Wellcome Trust 206250/Z/17/Z), Nikos I Bosse (Wellcome Trust 210758/Z/18/Z), Stefan Flasche (Wellcome Trust 208812/Z/17/Z), Rein M G J Houben (ERC \#757699), Simon R Procter (BMGF OPP1180644), Nicholas Davies (NIHR HPRU-2012-10096).

Funding: This research was funded by the European Union's Horizon 2020 research and innovation programme - project EpiPose No 101003688 (MJ, JE), National Institute for Health Research - Health Protection Research Unit in Immunisation (MJ) and for Modelling Methodology (MJ, TJ, JE), the Economic and Social Research Council RCUK grant ES/P010873/1 (TJ), the UK Public Health Rapid Support Team (TJ), the Bill and Melinda Gates Foundation grant OPP1183986 (ESN) the Nakajima Foundation (AE), the Alan Turing Institute (AE) and the Wellcome Trust grant 210758/Z/18/Z (SA). The views expressed in this publication are those of the author(s) and not necessarily those of the NIHR, Public Health England or the UK Department of Health and Social Care.

\section{Conflict of interest}

None declared.

Authors' contributions

Conceptualisation: MJ, WJE; model formulation: MJ, WJE, TJ, ESN, AE, SA; analysis and code development: MJ, TJ, ESN, $A E, S A$; writing: MJ, WJE, TJ, ESN, AE, SA. The members of the Working Group contributed equally in model formulation, analysis, writing reviewing the manuscript and approving the work for publication. The order was assigned randomly.

\section{References}

1. Public Health England (PHE). Coronavirus (COVID-19) in the UK. London: PHE. [Accessed: 3 Apr 2020]. Available from: https:// coronavirus.data.gov.uk/downloads/csv/coronavirus-cases_ latest.csv

2. Mizumoto K, Kagaya K, Zarebski A, Chowell G. Estimating the asymptomatic proportion of coronavirus disease 2019 (COVID-19) cases on board the Diamond Princess cruise ship, Yokohama, Japan, 2020. Euro Surveill. 2020;25(10). https:// doi.org/10.2807/1560-7917.ES.2020.25.10.2000180 PMID: 32183930

3. Public Health England (PHE). COVID-19 guidance and microbiology: fourth update. HPR volume 14 issue 6 (24 and 25 March). [Accessed: 28 April 2020]. Available from: https://www.gov.uk/government/ publications/health-protection-report-volume-14-2020/ hpr-volume-14-issue-6-24-and-25-march

4. Public Health England (PHE). COVID-19 Hospitalisation in England Surveillance System (CHESS) - daily reporting. [Accessed: 28 April 2020]. Available from: https://www. england.nhs.uk/coronavirus/wp-content/uploads/ sites/52/2020/03/phe-letter-to-trusts-re-daily-covid-19hospital-surveillance-11-march-2020.pdf

5. Verity R, Okell LC, Dorigatti I, Winskill P, Whittaker C, Imai N, et al. Estimates of the severity of coronavirus disease 2019: a model-based analysis. Lancet Infect Dis. 2020;0:S14733099(20)30243-7. https://doi.org/10.1016/S14733099(20)30243-7 PMID: 32240634

6. Bialek S, Boundy E, Bowen V, Chow N, Cohn A, Dowling N, et al. Severe outcomes among patients with coronavirus disease 2019 (COVID-19) - United States, February 12-March 16, 2020. MMWR Morb Mortal Wkly Rep. 2020;69(12):343-6. https://doi. org/10.15585/mmwr.mm6912e2 PMID: 32214079 
7. Riccardo F, Ajelli M, Andrianou X, Bella A, Manso M Del, Fabiani $M$, et al. Epidemiological characteristics of COVID-19 cases in Italy and estimates of the reproductive numbers one month into the epidemic. medRxiv. 2020;2020.04.08.20056861. doi: https://doi.org/10.1101/2020.04.08.20056861.

8. Nishiura H, Linton NM, Akhmetzhanov AR. Serial interval of novel coronavirus (COVID-19) infections. Int J Infect Dis. 2020;93:284-6. https://doi.org/10.1016/j.ijid.2020.02.060 PMID: 32145466

9. Zhou F, Yu T, Du R, Fan G, Liu Y, Liu Z, et al. Clinical course and risk factors for mortality of adult inpatients with COVID-19 in Wuhan, China: a retrospective cohort study. Lancet. 2020;395(10229):1054-62. https://doi.org/10.1016/S01406736(20)30566-3 PMID: 32171076

10. Lourenco J, Paton R, Ghafari M, Kraemer M, Thompson C, Simmonds $P$, et al. Fundamental principles of epidemic spread highlight the immediate need for large-scale serological surveys to assess the stage of the SARS-CoV-2 epidemic. medRxiv. 2020;2020.03.24.20042291. doi: https://doi. org/10.1101/2020.03.24.20042291.

11. British Medical Association (BMA). COVID-19 - ethical issues. A guidance note. London: BMA; 2020. Available from: https:// web.archive.org/web/20200426134240/https://www.bma.org. $\mathrm{uk} / \mathrm{media} / 2226 / \mathrm{bma-covid}$-19-ethics-guidance.pdf

\section{License, supplementary material and copyright}

This is an open-access article distributed under the terms of the Creative Commons Attribution (CC BY 4.0) Licence. You may share and adapt the material, but must give appropriate credit to the source, provide a link to the licence and indicate if changes were made.

Any supplementary material referenced in the article can be found in the online version.

This article is copyright of the authors or their affiliated institutions, 2020. 